Hamamatsu, the Department of Pediatrics, National Rehabilitation Center for Disabled Children, Tokyo, and the Department of Pediatrics, Nagoya City University Medical School, Japan. Perinatal asphyxia had been diagnosed in 16 , neonatal jaundice in 2 , and no predisposing condition in 4 . MRI lesions possibly caused by asphyxia were found in the basal ganglia, thalamus, and/or cerebral white matter in 14 of 16 children. No abnormalities were seen in the cerebral cortex of the subjects. Six children with lesions in both the thalamus and putamen had moderate or severe motor abnormality. Children with lesions in the cerebral white matter only had mild motor abnormality. (Yokochi $\mathrm{K}$ et al. Magnetic resonance imaging in athetotic cerebral palsied children. Acta Paediatr Scand Sept 1991; $\underline{80: 818-823 .) ~(C o r r e s p: ~ D r . ~ Y o k o c h i, ~}$ Seirei-Mikatabara Gen. Hosp., Mikatabara 3453, Hamamatsu, Shizuoka 433, Japan.)

COMMENT. Brain lesions causing athetosis in CP children may not be revealed by $\mathrm{CT}$ scan but high intensity areas in the thalamus, putamen and/or cerebral white matter may be demonstrated in T2weighted images of the MRI. The evidence of perinatal asphyxia in 16 of the 22 children reported was judged by Apgar scores of 3 and below, generalized hypotonia, and convulsions in the neonatal period. The MR imaging was performed at 2-12 years of age and studies obtained soon after birth might have documented a prenatal timing for the lesions.

\title{
NEONATAL CSF CREATINE KINASE AND CP RISK
}

Creatine kinase brain isoenzyme (CK-BB) was examined in the cerebrospinal fluid of 150 neonates at the Departments of Pediatrics and Neonatal Medicine and Clinical Chemistry, University Hospital, Ghent, Belgium. Indications for lumbar puncture were intraventricular hemorrhage, subarachnoid hemorrhage, postasphyxial encephalopathy, convulsions, birth trauma, periventricular echodensities, or question of sepsis. Newborns with a neurologic disorder showed significantly higher concentrations of immunoreactive CK-BB than did normal newborns or those with subarachnoid hemorrhage. (The median $\mathrm{CK}-\mathrm{BB}$ for 5 neonates with intraventricular hemorrhage and echodensities was $106 \mathrm{mcg} / \mathrm{l}$ and the normal control was 2.14.) An independent effect of seizures on the CK-BB concentration was not documented. High concentrations of CSF CK-BB in the neonatal period were correlated with an adverse outcome - that is death or abnormal neurologic exam at discharge. (De Praeter C et al. Creatine kinase isoenzyme BB concentrations in the cerebrospinal fluid of newborns: relationship to short-

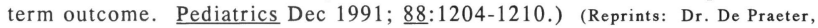
Dept. of Pediatrics, University Hospital, Ghent, De Pintelaan, 185, B-9000, Ghent, Belgium.)

COMMENT. Determination of the CK-BB concentration in CSF appears to be a clinically useful test for the prediction of neurologic disorders in high risk neonates. Infants with high CSF CK-BB concentrations should receive careful follow-up. In a previous study (Fernandez F et al. Acta Paediatr Scand 1987; $\underline{76}: 914$ ) an elevated serum CPK measured within 4 hours after birth was a sensitive indicator of brain damage in asphyxiated term infants but was of limited 
prognostic value in assessment of neurological outcome. (See Progress in Pediatric Neurology, Millichap JG ed, 1991, page 333)

Cerebro spinal fluid examination in symptom-free infants with risk factors for infection was evaluated in 284 newborns at the Department of Pediatrics, Rush Presbyterian-St. Luke's Medical Center, Chicago, IL (Fielkow S et al. J Pediatr Dec 1991; 119:971-973). Positive cultures without pleocytosis in 5 infants $(1.8 \%)$ were contaminants and none of the symptom-free infants had meningitis. The authors conclude that CSF examination is not indicated in the diagnostic evaluation of symptom-free infants born to mothers with chorioamnionitis or other risk factors for neonatal infection.

\section{NEUROCUTANEOUS SYNDROMES}

\section{VISUAL LOSS IN TUBEROUS SCLEROSIS}

Visual loss in 4 patients with tuberous sclerosis complicated by subependymal giant-cell astrocytoma is reported from the Departments of Ophthalmology, Neurology, and Radiology, University of Michigan Medical Center, Ann Arbor, MI. The patients presented at 12-20 years of age with obstructive hydrocephalus. Surgery relieved elevated pressure in all cases but 2 patients became blind and 1 has severe visual field loss from the effects of chronic papilledema on the optic nerves. Early surgical decompression prevented visual loss in the fourth patient whose tumor was removed at 12 years of age and who required further resection of a giant-cell astrocytoma at 20 years. (Dotan, SA et al. Visual loss in tuberous sclerosis. Neurology Dec 1991; 41:1915-1917.) (Reprints: Dr. J.D. Trobe, W.K. Kellogg Eye Center, 1000 Wall St., Ann Arbor, MI 48105.)

COMMENT. Periodic opthamologic examination and brain imaging are advisable in tuberous sclerosis patients with subependymal nodules. The timely relief of increased intracranial pressure may arrest or prevent loss of vision.

\section{FAMILIAL SPINAL NEUROFIBROMATOSIS}

The clinical features and genetic linkage analysis of two pedigrees with familial spinal neurofibromatosis (NF) are described from the Divisions of Neurology and Medical Genetics, Cedars-Sinai Medical Center, UCLA School of Medicine, Los Angeles, CA; the Neurofibromatosis Institute, Pasadena, $\mathrm{CA}$; and Department of Medical Informatics, University of Utah, Salt Lake City, UT. On clinical grounds, it was difficult to assign the two families to NF1 or NF2 based on the criteria established by the National Institutes of Health. Cutaneous tumors, Lisch nodules or acoustic tumors were absent. Cafe-au-lait spots were present in 1 family and absent in the other. The inheritance pattern was autosomal dominant in both pedigrees. Genetic linkage analysis was performed with markers linked to the NF1 gene on chromosome 17 and markers linked to the NF2 gene on chromosome 22. The 